\title{
Docosahexaenoic Acid Enhances Cyclooxygenase-2 Induction by Facilitating p44/42, but Not p38, Mitogen-Activated Protein Kinase Activation in Rat Vascular Smooth Muscle Cells
}

\author{
Takuji Machida $^{1}$, Miho Hiramatsu ${ }^{1}$, Naoya Hamaue ${ }^{1}$, Masaru Minami ${ }^{1}$, and Masahiko Hirafuji ${ }^{1, *}$ \\ ${ }^{1}$ Department of Pharmacology, Faculty of Pharmaceutical Sciences, Health Sciences University of Hokkaido, \\ Ishikari-Tobetsu, Hokkaido 061-0293, Japan
}

Received April 14, 2005; Accepted July 19, 2005

\begin{abstract}
The effect of docosahexaenoic acid (DHA) on cyclooxygenase expression induced by interleukin (IL)-1 $\beta$ and phorbol 12-myristate 13-acetate (PMA) in rat vascular smooth muscle cells (VSMCs) was investigated in order to clarify the cellular mechanism of cardiovascular protective effects. DHA and eicosapentaenoic acid slightly enhanced IL- $1 \beta$-induced cyclooxygenase (COX)-2, but not COX-1, expression, whereas arachidonic acid had no effect. DHA also slightly enhanced PMA-induced COX-2 expression. DHA stimulated both rapid and prolonged activation of $\mathrm{p} 44 / 42$, but not $\mathrm{p} 38$, mitogen-activated protein kinase (MAPK) induced by IL- $1 \beta$ and PMA. These results suggest that DHA enhances the COX-2 expression by selectively facilitating p44/42 MAPK activation in VSMCs.
\end{abstract}

Keywords: docosahexaenoic acid, cyclooxygenase-2, vascular smooth muscle cell

It is well known that n-3 polyunsaturated fatty acids such as eicosapentaenoic acid (EPA) and docosahexaenoic acid (DHA), active components of fish oils, have several cardiovascular protective effects (1). Although the precise cellular and molecular mechanisms are not yet fully understood, these protective effects could be partially attributed to their direct effects on vascular smooth muscle cell (VSMC) functions. Indeed, these fatty acids have been shown to improve vascular contractility and remodeling and to favorably alter eicosanoid profile and inducible nitric oxide synthase activity $(1,2)$.

Cyclooxygenese $(\mathrm{COX})$ is the rate-limiting enzyme for the conversion of arachidonic acid to various prostaglandins (PGs) and thromboxane. There are two isoforms of $\mathrm{COX}$, that is, constitutive COX-1 and inducible COX-2. These COX expressions and prostaglandin $\mathrm{I}_{2}\left(\mathrm{PGI}_{2}\right)$ release in the vascular wall has important physiological and pathological roles in the cardiovascular system (3). Therefore, there is a possibility that the mechanism for cardioprotective effects of DHA is, at least in part, attributable to the

*Corresponding author. FAX: +81-0133-23-1370

E-mail: hirafuji@hoku-iryo-u.ac.jp influence on COX-1 and COX-2 expression in VSMCs as well as vascular endothelial cells. COX-2 induction and $\mathrm{PGI}_{2}$ release are stimulated with interleukin (IL)-1 $\beta$ or phorbol 12-myristate 13-acetate (PMA) in various cells including VSMCs (4-6). Mitogen-activated protein kinase (MAPK) cascades including p44/42 and/or p38 MAPK are involved in the mechanism of IL- $1 \beta$ - and PMA-induced COX-2 expression in VSMCs $(4-6)$. The aim of the present study was, therefore, to evaluate the effect of DHA on COX-1 and COX-2 expression and MAPK activity in rat VSMCs.

VSMCs were enzymatically isolated from aortic media of 6-7-week-old Wistar Kyoto rats and cultured in Dulbecco's modified Eagle medium containing 10\% fetal bovine serum (Life Technologies, Grand Island, NY, USA), as described previously (7). After $6-8$ days culture, the sub-confluent cells were incubated in serumfree medium containing $0.1 \%$ bovine serum albumin (BSA) for $24 \mathrm{~h}$ before experiments. Cells were then treated with IL- $1 \beta$ (Collaborative Biomedical Products, Bedford, MA, USA) or PMA (Biomol Research Laboratories, Plymouth Meeting, PA, USA) in the presence of polyunsaturated fatty acids (Sigma, St. Louis, MO, USA). These fatty acids were dissolved as their respective sodium salt in physiological saline 
containing $0.1 \%$ BSA as a carrier protein and then further diluted with the serum-free culture medium.

Protein expression was evaluated by Western blotting and densitometic analysis as described previously (7). COX-1 and COX-2 proteins were detected with COX-1 and COX-2 polyclonal rabbit antiserum (Cayman Chemical, Ann Arbor, MI, USA), respectively. Activation of MAPK was evaluated by analyzing the phosphorylation of $\mathrm{p} 44 / 42$ and $\mathrm{p} 38$ MAPK proteins, using phospho-p44/42 MAPK and phospho-p38 MAPK polyclonal rabbit antiserum (Promega, Madison, WI, USA), respectively.

Results were expressed as the mean \pm S.E.M. of replicate experiments. Statistical analysis of the results was made by Student's $t$-test.

Figure $1 \mathrm{~A}$ shows the effect of DHA on COX-1 and COX-2 expressions induced by IL- $1 \beta$. IL- $1 \beta(3 \mathrm{ng} / \mathrm{ml})$ stimulation for $24 \mathrm{~h}$ induced COX-2 expression, which was further enhanced by the co-incubation with DHA in a concentration-dependent manner. Although a slight COX-2 protein expression was detected in the unstimu- lated cells, DHA alone had no significant effect (data not shown). In contrast, IL-1 $\beta$ or DHA had no effect on COX-1 expression. Figure 1B summarizes the effects of DHA and EPA (30 $\mu \mathrm{M}$ each) on COX-2 expression induced by IL-1 $\beta$. DHA and EPA slightly but significantly increased the COX-2 expression to $142.3 \pm 18.4 \%$ and $132.6 \pm 10.4 \%$ of the control, $\mathrm{n}=9$ and 7 , respectively. However, arachidonic acid $(30 \mu \mathrm{M})$ had no significant effect $(126.8 \pm 14.6 \%$ of the control, $\mathrm{n}=7)$.

Figure 1C shows the effect of DHA on $\mathrm{p} 44 / 42$ and p38 MAPK activation induced by IL- $1 \beta(3 \mathrm{ng} / \mathrm{ml})$. As shown in Fig. 1D, DHA $(30 \mu \mathrm{M})$ slightly but significantly enhanced the IL- $1 \beta$-induced p44/42 MAPK activation after 12-h stimulation, while it had no effect on the p38 MAPK activation, as measured by immunodetection of its phosphorylation. DHA alone had no significant effect without IL- $1 \beta$ stimulation (data not shown).

Figure 2A shows the effect of DHA on COX-2 expression induced by PMA. PMA stimulation for $4 \mathrm{~h}$ induced the COX-2 protein expression in a concentra-
(A)

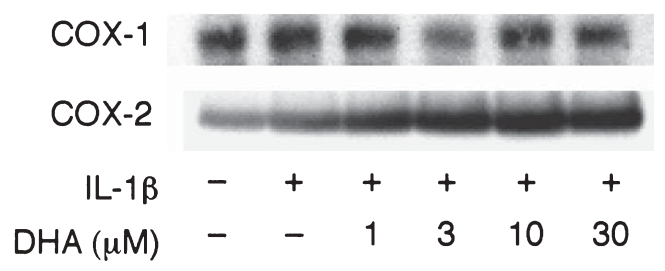

(B)
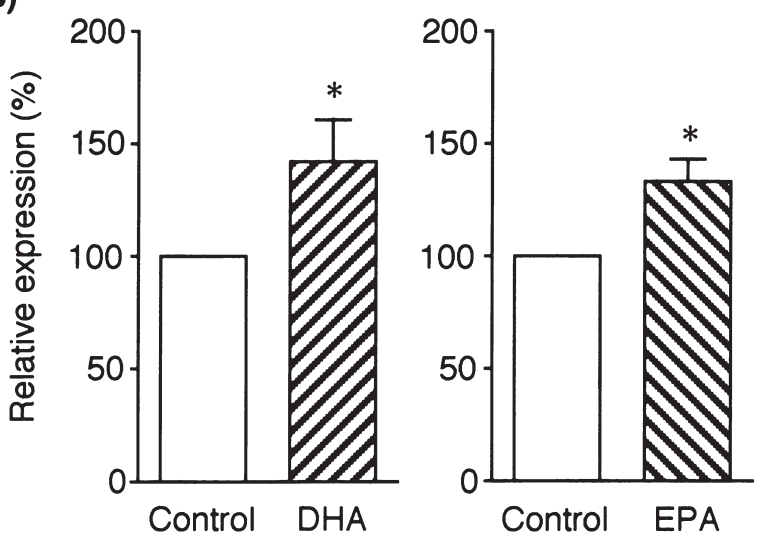

(C)

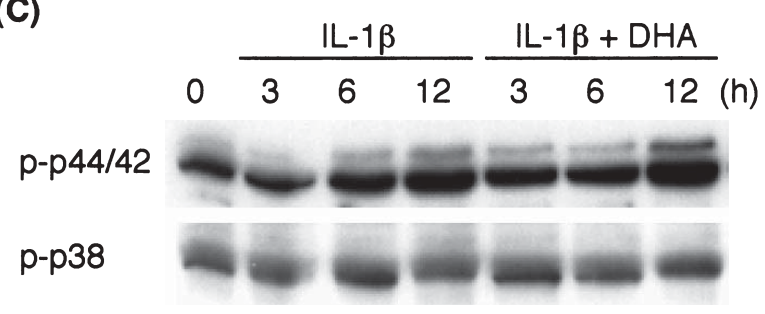

(D)

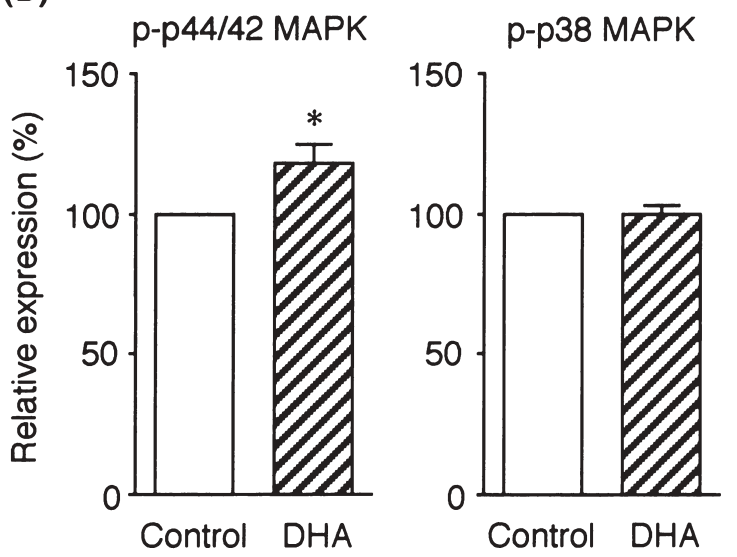

Fig. 1. Enhancement of IL- $1 \beta$-induced COX-2 expression and p44/42 MAPK activation by $30 \mu \mathrm{M}$ DHA. A: Effect of DHA on COX-1 and COX-2 protein expression. Cells were stimulated with $3 \mathrm{ng} / \mathrm{ml} \mathrm{IL}-1 \beta$ for $24 \mathrm{~h}$. B: Summary of densitometric analysis showing the effects of DHA and EPA (30 $\mu \mathrm{M}$ each) on IL- $1 \beta$-induced COX-2 protein expression. Cells were stimulated with IL- $1 \beta$ for $24 \mathrm{~h}$. Results are each the mean \pm S.E.M. of 9 and 7 experiments, respectively, compared with the control (IL- $1 \beta$ alone). C: Effects of DHA on IL-1 $\beta$-induced phosphorylation of p44/42 (p-p44/42, upper) and p38 (p-p38, lower) MAPK. Cells were stimulated with IL- $1 \beta$ for the indicated time periods in the presence of DHA. D: Summary of densitometric analysis showing the effect of DHA on p44/42 (left) and p38 (right) MAPK activation after 12-h stimulation. Results are each the mean \pm S.E.M. of 3 experiments compared with each control (IL- $1 \beta$ alone). $* P<0.05$ versus control. 
tion-dependent manner, which was further enhanced by $30 \mu \mathrm{M}$ DHA. DHA slightly but significantly enhanced PMA (10 nM)-induced COX-2 expression to $123.0 \pm$

(A)

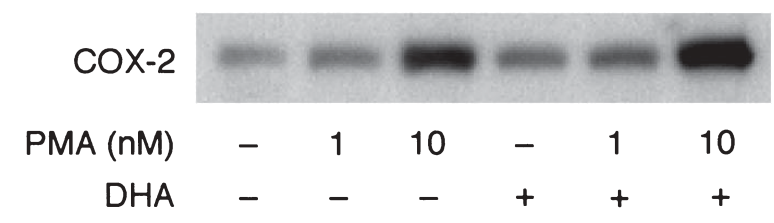

(B)

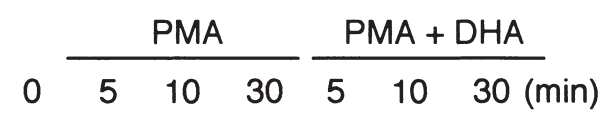

p-p44/42

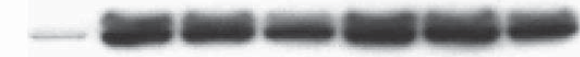

p-p38

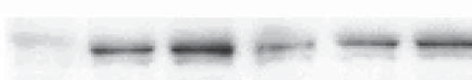

(C)

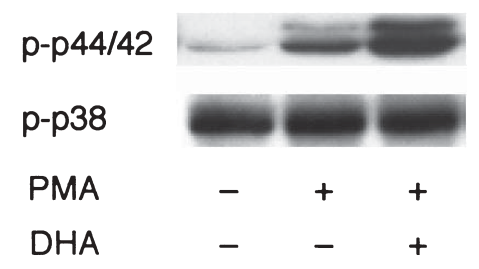

(D)

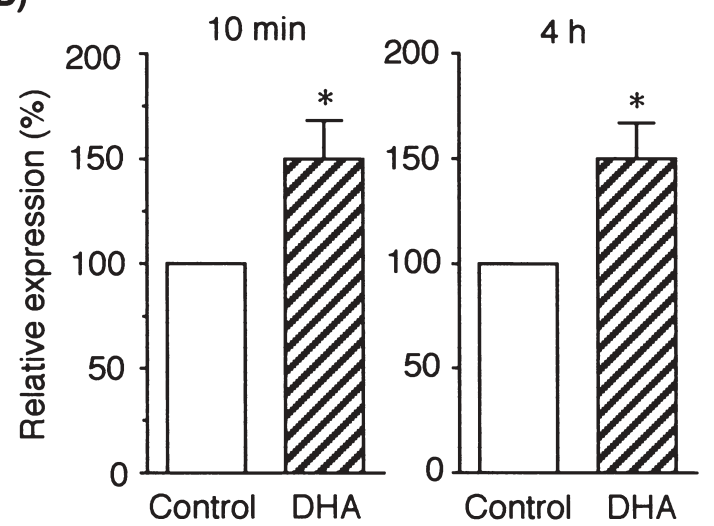

Fig. 2. Enhancement of PMA-induced COX-2 expression and p44/42 MAPK activation by $30 \mu \mathrm{M}$ DHA. A: Effect of DHA on PMA-induced COX-2 expression. Cells were stimulated with PMA for $4 \mathrm{~h}$. B: Effect of DHA on p44/42 (p-p44/42, upper) and p38 (p-p38, lower) MAPK phosphorylation induced by short term exposure to PMA. Cells were stimulated with PMA for the indicated time periods up to $30 \mathrm{~min}$ in the presence of DHA. C: Effect of DHA on p44/42 (p-p44/42, upper) and p38 (p-p38, lower) MAPK phosphorylation induced by 4 -h exposure to PMA. Cells were stimulated with PMA for $4 \mathrm{~h}$ in the presence of DHA. D: Summary of densitometric analysis showing the effect of DHA on p44/42 MAPK activation after 10-min (left) and 4-h (right) stimulation. Results are each the mean \pm S.E.M. of 4 experiments compared with each control (PMA alone). ${ }^{*} P<0.05$ versus control.
$7.1 \%$ of the control $(n=5)$. PMA caused a rapid and persistent activation of p44/42 MAPK and a transient activation of p38 MAPK. Figure $2 \mathrm{~B}$ and $2 \mathrm{C}$ show the effect of DHA on p44/42 and p38 MAPK activation induced by exposure to PMA $(10 \mathrm{nM})$ for short term up to $30 \mathrm{~min}$ and for $4 \mathrm{~h}$, respectively. As summarized in Fig. 2D, DHA $(30 \mu \mathrm{M})$ significantly enhanced the p44/42 MAPK activation induced by not only $10 \mathrm{~min}$ (left) but also $4 \mathrm{~h}$ (right) exposure to PMA, while again it had no effect on the p38 MAPK activation.

The present study for the first time demonstrated that DHA enhanced IL- $1 \beta$-induced COX- 2 expression in VSMCs. There was no significant effect of DHA on COX-1 expression. The enhancing effect of DHA specific to COX-2 induction was small but reproducible. EPA, but not arachidonic acid, also showed a similar effect, suggesting the common feature of n-3 polyunsaturated fatty acids.

Activation of p44/42 and/or p38 MAPK are involved in IL- $1 \beta$-induced COX-2 expression in smooth muscle cells $(5,8)$. Consistent with these reports, we confirmed that both PD 98059, a p44/42 MAPK kinase inhibitor, and SB 203580, a p38 MAPK inhibitor, partially inhibited and their combination additively inhibited the IL- $1 \beta$-induced COX-2 expressions (data not shown). Although cells were incubated for $24 \mathrm{~h}$ in serum-free culture medium to render them quiescent before the experiment, a slight COX-2 expression was detected in the unstimulated cells. This may be due to activation of p38 MAPK by serum starvation (9), which may explain little effect of IL- $1 \beta$ on p38 MAPK in our study. IL- $1 \beta$ induces a rapid and transient activation of $\mathrm{p} 44 / 42$ MAPK, followed by the prolonged activation in VSMCs (10). COX-2 expression is dependent on the prolonged activation of $\mathrm{p} 44 / 42$ MAPK and the subsequent activation of nuclear factor $-\kappa \mathrm{B}(5)$. We previously demonstrated that DHA enhanced the transient IL- $1 \beta$-induced activation of $\mathrm{p} 44 / 42$ MAPK (7). The present study demonstrated that DHA also enhanced the prolonged activation of $\mathrm{p} 44 / 42$ MAPK, while it had no effect on the p38 MAPK activation. Similar results were obtained with a phorbol ester, PMA. PMA induces COX-2 expression through $\mathrm{p} 44 / 42$ and $\mathrm{p} 38$ MAPK activation in many cell types including VSMCs $(3,4,11)$. The present study clearly showed that DHA also slightly but reproducibly enhanced PMA-induced COX-2 expression, and stimulated both rapid and prolonged activation of p44/42 MAPK, while it had no effect on the p38 MAPK activation. These results suggest that DHA enhanced the COX-2 induction by selectively facilitating the activation of $\mathrm{p} 44 / 42$ MAPK in VSMCs.

Several studies have investigated the effects of DHA and EPA on COX-2 expression and MAPK activity 
using various cell types including VSMCs. However, highly conflicting results were obtained: both enhancement (12) and inhibition (13) of COX-2 induction have been reported. Similarly, both enhancement $(7,14)$ and inhibition (13) of p44/42 or p38 MAPK have been reported. Reasons for these discrepancies are currently unclear, but may be explained by cell type- or stimulusspecific signaling mechanisms involved in the COX-2 induction or by experimental conditions such as concentration and applied form of fatty acids.

Importently, it has been reported that the long term inhibition of COX-2 by the selective COX-2 inhibitor causes the increased incidence of myocardial infarction and stroke in the placebo controlled trials (15). This result can be explained by the suppression of the atheroprotective role of COX-2 expressed in endothelial cells, since the predominantly released $\mathrm{PGI}_{2}$ is considered to be antiatherogenic, causing vasodilation, platelet and leukocyte inhibition, and inhibition of VSMC proliferation (3). COX-2 is also induced in VSMCs by stimulation with various growth factors and cytokines, causing predominantly cardioprotective $\mathrm{PGI}_{2}$ release $(4-6)$, although the pathophysiological role is still unclear. Administration of fish oils rich in DHA and EPA has been shown to increase the production of $\mathrm{PGI}_{2}$ and $\mathrm{PGI}_{3}$ which has equipotent biological activities with $\mathrm{PGI}_{2}$, in several human studies and animal experiments in vivo (1). It may be explained in part by the augmentation of endothelial COX-2 expression by DHA (12). The present study demonstrated that DHA also mildly enhanced the COX-2 induction in VSMCs. On the contrary, DHA is reported to inhibit the COX-2 induction in macrophages which are predominantly releasing proatherogenic $\mathrm{PGE}_{2}$ (13). Therefore, there is a possibility that the mild and persistent enhancement of COX-2 expression in VSMCs by DHA may also contribute to the cardioprotective effects, probably by releasing $\mathrm{PGI}_{2} / \mathrm{PGI}_{3}$ as a compensatory mechanism for the endothelial dysfunction. Further studies are still required in order to clarify the pathophysiological relevance of our finding as well as the precise mechanism of selective facilitation of $\mathrm{p} 44 / 42$ MAPK activation by DHA.

\section{Acknowledgments}

This work was supported in part by Grant-in-Aid for Scientific Research from Japan Society for the Promotion of Science (No. 14572061) and the Academic Science Frontier Project of the Ministry of Education, Culture, Sports, Science, and Technology, Japan.

\section{References}

1 Abeywardena MY, Head RJ. Longchain n-3 polyunsaturated fatty acids and blood vessel function. Cardiovasc Res. 2001;52: 361-371.

2 Hirafuji M, Machida T, Hamaue N, Minami M. Cardiovascular protective effects of $n-3$ polyunsaturated fatty acids with special emphasis on docosahexaenoic acid. J Pharmacol Sci. 2003;92: 308-316.

3 Vane JR. Back to an aspirin a day? Science. 2002;296:474-475.

4 Baillie G, Mackenzie SJ, Houslay MD. Phorbol 12-myristate 13acetate triggers the protein kinase A-mediated phosphorylation and activation of the PDE4D5 cAMP phosphodiesterase in human aortic smooth muscle cells through a route involving extracellular signal regulated kinase (ERK). Mol Pharmacol. 2001;60:1100-1111.

5 Frias MA, Dubouloz F, Rebsamen MC, Lang U. Prostacyclin production in rat aortic smooth muscle cells: role of protein kinase $\mathrm{C}$, phospholipase $\mathrm{D}$ and cyclooxygenase-2 expression. Cardiovasc Res. 2003;60:438-446.

6 Jiang B, Xu S, Hou X, Pimental DR, Brecher P, Cohen RA. Temporal control of NF- $\kappa \mathrm{B}$ activation by ERK differentially regulates interleukin-1 $\beta$-induced gene expression. J Biol Chem. 2004;279:1323-1329.

7 Hirafuji M, Machida T, Tsunoda M, Miyamoto A, Minami M. Docosahexaenoic acid potentiates interleukin- $1 \beta$ induction of nitric oxide synthase mechanism through involving p44/42 MAPK activation in rat vascular smooth muscle cells. Br J Pharmacol. 2002;136:613-619.

8 Yang CM, Chien CS, Hsiao LD, Luo SF, Wang CC. Interleukin$1 \beta$-induced cyxlooxygenase- 2 expression is mediated through activation of $\mathrm{p} 42 / 44$ and $\mathrm{p} 38$ MAPKS, and NF- $\kappa$ B pathways in canine tracheal smooth muscle cells. Cell Signal. 2002;14:899911.

9 Kummer JL, Rao PK, Heidenreich KA. Apoptosis induced by withdrawal of trophic factors is mediated by p38 mitogenactivated protein kinase. J Biol Chem. 1997;272:20490-20494.

10 Jiang B, Brecher P, Cohen RA. Persistent activation of nuclear factor- $\kappa \mathrm{B}$ by interleukin- $1 \beta$ and subsequent inducible NO synthase expression requires extracellular signal-regulated kinase. Arterioscler Thromb Vasc Biol. 2001;21:1915-1920.

11 Schuette R, LaPointe MC. Phorbol ester stimulates cyclooxygenase- 2 expression and prostanoid production in cardiac myocytes. Am J Physiol Heart Circ Physiol. 2000;279:H719H725.

12 Gilbert M, Dalloz S, Maclouf J, Lagarde M. Differential effects of long chain n-3 fatty acids on the expression of PGH synthase isoforms in bovine aortic endothelial cells. Prostaglandins Leukot Essent Fatty Acids. 1999;60:363-365.

13 Lee JY, Plakidas A, Lee WH, Heikkinen A, Chanmugam P, Bray G, et al. Differential modulation of toll-like receptors by fatty acids: preferential inhibition by $n-3$ polyunsaturated fatty acids. J Lipid Res. 2003;44:479-486.

14 Diep QN, Touyz RM, Schiffrin EL. Docosahexaenoic acid, a peroxisome proliferator-activated receptor- $\alpha$ ligand, induces apoptosis in vascular smooth muscle cells by stimulation of p38 mitogen-activated protein kinase. Hypertension. 2000;36: 851-855.

15 Bombardier C, Laine L, Reicin A, Shapiro D, Burgos-Vargas $\mathrm{R}$, Davis B, et al. Comparison of upper gastrointestinal toxicity of rofecoxib and naproxen in patients with rheumatoid arthritis. N Engl J Med. 2000;343:1520-1528. 